\title{
RESEARCH ON THE CONTROL OF REACTIVE POWER COMPENSATION BASED ON FUZZY REASONING
}

\author{
Ping Yang ${ }^{1}$, Yuwen Sun ${ }^{2}$, Jianguo Dong ${ }^{3}$, Yong Yang ${ }^{1, *}$ \\ I School of Information and Electrical Engineering, Shenyang Agricultural University, \\ Shenyang, Liaoning Province, P. R. China 110161 \\ ${ }^{2}$ School of Information Science and Technology, Nanjing Agricultural University, Nanjing, \\ Jiangsu Province, P. R. China 210095 \\ ${ }^{3}$ School of Sciences, Shenyang Agricultural University, Shenyang, Liaoning Province, P. R. \\ China 110161 \\ * Corresponding author, Address: School of Information and Electrical Engineering, \\ Shenyang Agricultural University, 120 Dongling Road, Shenyang110161, Liaoning \\ Province, P. R. China, Tel:+86-24-88487860, Fax:+86-24-88487122, Email:xdxy7128 \\ (a) $126 . c o m$
}

\begin{abstract}
With the development of the rural power grids increasing gradually, there are higher requirements needed for its operation in reliability and economy. Reactive compensation using shunt capacitor at both ends of the load, reducing current of lines, and improves the power factor of system. The objective is to reduce losses of line, and improves the quality of voltage and the utilization of electrical equipments. In the switching control of compensation device, using voltage and reactive power these two factors to control, this is the fuzzy control. The range of voltage and reactive power are divided into several regions to determine the membership of each region. Using MATLAB simulation achieves a design method of fuzzy reasoning logic control, and gets an ideal control results.
\end{abstract}

Keywords: fuzzy reasoning, reactive power compensation, method of control, MATLAB simulation

Please use the following format when citing this chapter:

Yang, P., Sun, Y., Dong, J. and Yang, Y., 2009, in IFIP International Federation for Information Processing, Volume 293, Computer and Computing Technologies in Agriculture II, Volume 1, eds. D. Li, Z. Chunjiang, (Boston: Springer), pp. 743-750. 


\section{INTRODUCTION}

With the growing scale grid, its economy and reliability requirements are also getting higher and higher. Improving the power grid operation quality and power factor grid and reducing network loss are a very important work (Wang Chun et al., 2008). In fact, the existence of a large number emotional load, reduces the power factor greatly, increases loss of active lines in result, lowers users voltage, makes the power equipment not fully applied and undermines the stability of the power system, and other adverse conditions (Wu Gongwen et al., 2008; Yu Haisheng et al., 2000). When a serious lack of reactive power collapses the entire system, a rational approach is reactive power compensation device installed where necessary reactive power consumption needed (E Zhijun et al., 2008; Liu Liming et al., 2002).

\section{THE PRINCIPLE AND PURPOSE OF REACTIVE COMPENSATION}

Reactive power compensation is the substance of the circuit to reduce the inductive reactive power, commonly used method is the inductive load paralleled with an appropriate capacitor, as shown in Fig. 1 (Gao Bo et al., 2003).

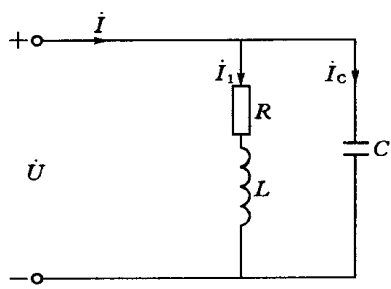

(a)

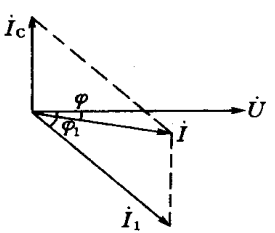

(b)

Fig. 1: The principle of reactive power compensation

(a) Equivalent circuit of reactive power compensation (b) Reactive compensation vectors

Shunt capacitor $C$, circuit current $i=i_{1}$, angle $\varphi_{1}$ lagging behind voltage.

Shunt capacitor $C$, circuit current $i=i_{1}+i_{C}, \varphi$ decreased, but the power factor $\cos \varphi$ will be changed bigger, thereby, the entire circuit of the power factor will be improved too (Liu Shumin et al., 2002).

Reactive power compensation or improving power factor in network has a very important practical significance and economic benefits in reducing line losses, improving the voltage quality, and increasing efficiency in the use of electrical equipment (E. M. John, 2002; Xiong Hugang et al., 2008). 
(1) Reducing power losses in power grids and power loss

When the active power $\mathrm{P}$ fixed in value, and $\cos \varphi$ inversely proportional to load current $\mathrm{I}$, and reactive power compensation equipment installed by the formula $\mathrm{I}=\mathrm{P} /(\mathrm{U} \cdot \cos \varphi)$, power factor $\cos \varphi$ will be improved to decreases the current line, so that line loss $\Delta \mathrm{P}$ will be lower:

$\Delta \mathrm{P}=\mathrm{I}^{2} \mathrm{R}$. Therefore, the main purpose of installing reactive compensation equipment is to reduce line losses in the power system (Mao Meijun, 2007).

(2) Improving the voltage quality

Calculation formula for the line voltage loss $\Delta \mathrm{U}$ :

$$
\begin{gathered}
\Delta \mathrm{U}=\frac{P R \quad+X}{U_{e}} \\
\Delta \mathrm{U} \text { - line voltage loss } \mathrm{kV} \\
\mathrm{P} \text { _-active power MW } \\
\mathrm{Q} \text {-reactive power } \mathrm{MVar} \\
\mathrm{Ue} \text {-rated voltage } \mathrm{kV} \\
\mathrm{R} \text { - lines of resistance } \Omega \\
\mathrm{X} \text { - line inductive reactance } \Omega
\end{gathered}
$$

From that, when the line of reactive power $\mathrm{Q}$ reduced the voltage loss

$\Delta \mathrm{U}$ also has been reduced (Cai Zhongqin et al., 1998).

(3) Increasing the power supply capacity equipment

From the $\mathrm{P}=\mathrm{S} \cdot \cos \varphi$, when the apparent power equipment $\mathrm{S}$ certain, power factor $\cos \varphi$ improved, $\mathrm{P}$ in last formula will also be increased, electrical equipment meritorious effort will also be increased too (Tang Yong et al., 1995).

(4) Reducing Users electricity costs, lowering production costs, and avoiding the low power factor off the electricity sector fine (P. Douglas et al., 1999).

\section{THE METHOD AND REALIZATION OF FUZZY CONTROL}

Generally, reactive compensation of electrical net adopts automatic switching device, they are deserved to in-depth study, how to control toand-fro switching problem of compensation device, which is the best control method (Xu Yonghai et al., 2006). Fuzzy mathematics is a subject which researches and disposes fuzzy phenomenon (Liu Xiaochuan et al., 2000). It can't use classical mathematics to generally estimate and control electrical net's state which is a fuzzy concept. 
It is a best method to carry through general fuzzy estimate with applying fuzzy discursion in control capacitor's switching device.

According to simulation of MATLAB and the control rules of voltage, five fuzzy subsets reflect deviation with voltage deviation as input variable, that is, NO(Normal), LO(Low), MI(Minimum), HI(high), MA(Maximum), fuzzy domain is $[-2,2]$; With reactive power deviation $\Delta c$ as input, nine fuzzy subsets reflect deviation, that is, $\mathrm{NH}$ (negative high), NS(negative small ), NM(negative mediate), NB(negative big), NO(zero), PS(positive small), $\mathrm{PM}$ (positive mediate), $\mathrm{PB}$ (positive big), $\mathrm{PH}$ (positive high ), fuzzy domain is $[-4,4]$.

Fig.2 and Fig.3 respectively descript membership function of voltage deviation and reactive power deviation with simulation of MATLAB.

Membership function converses the actual variable to linguistic variables, and determines membership of discrete fuzzy sets in linguistic value.

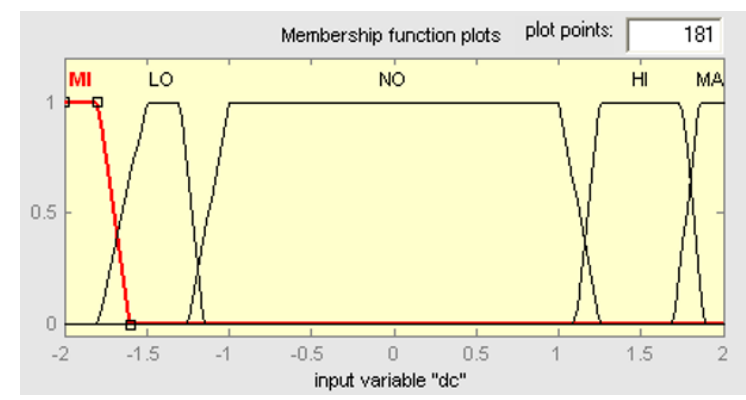

Fig.2: The membership function of $\Delta u$

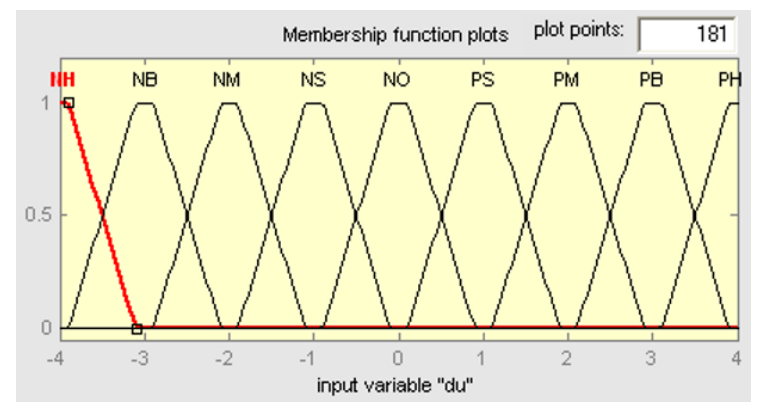

Fig.3: The membership function of $\Delta c$

Fuzzy ruling is described by nine fuzzy subsets, that is, NS(negative small), NM(negative mediate), NB(negative big), NA(negative all), ST(stable), PS((positive small), PM((positive mediate), PB(positive big), PA(positive all), fuzzy domain is [-7, 7]. Membership function is described by Fig.4. 


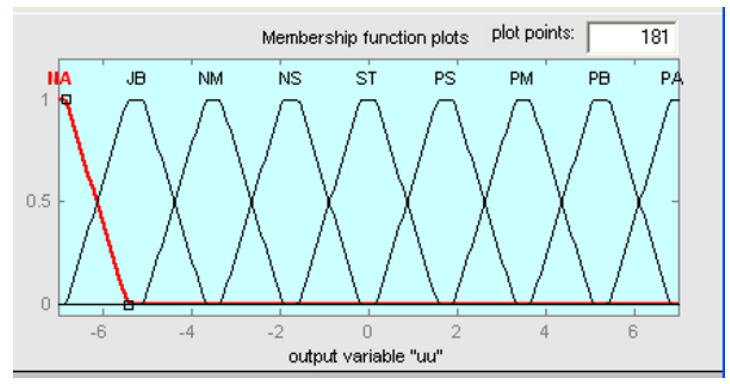

Fig.4: The membership function of $\mathrm{U}$

Inference rules following the below are obtained by the debugging in scene and experience reactive power compensation.

If $\Delta u$ is NO and $\Delta c$ is NS, Control Variable U is PS;

If $\Delta u$ is $\mathrm{NO}$ and $\Delta c$ is $\mathrm{NM}$, Control Variable $\mathrm{U}$ is $\mathrm{PM}$;

If $\Delta u$ is $\mathrm{NO}$ and $\Delta c$ is $\mathrm{NB}$, Control Variable $\mathrm{U}$ is $\mathrm{PB}$;

Fuzzy state control table is described by table, according to 35 fuzzy reasoning language rules, as the following table:

Table 1. The general table of Fuzzy control rule

\begin{tabular}{|c|c|c|c|c|c|c|c|c|c|}
\hline$\frac{\triangle \mathrm{C}}{\triangle \mathrm{U}}$ & $\mathrm{NH}$ & NB & NM & NS & $\mathrm{ZE}$ & PS & PM & PB & PH \\
\hline MI & NA & NA & NA & NA & NA & NA & NA & $\mathrm{NA}$ & NA \\
\hline LO & ST & ST & ST & ST & - & - & - & - & - \\
\hline NO & PA & PB & $\mathrm{PM}$ & PS & ST & NS & NM & NB & NA \\
\hline HI & - & - & - & - & - & ST & ST & ST & ST \\
\hline MA & NA & NB & NM & NS & NS & NS & NS & NS & NS \\
\hline
\end{tabular}

Each control rules can be obtained corresponding fuzzy relations, as $\widetilde{R}_{1}, \widetilde{R}_{2}, \cdots, \widetilde{R}_{35}$. So the corresponding fuzzy relationship of the entire system control rules is

$$
\widetilde{R}=\widetilde{R}_{1} \vee \widetilde{R}_{2} \vee \cdots \vee \widetilde{R}_{35}=\vee_{i=1}^{35} \widetilde{R}_{i}
$$

The fuzzy sets of corresponding control $\widetilde{U}$ as follows can be obtained by membership function of $\Delta u$ and $\Delta c$ after getting overall fuzzy relationship ,not from the principle of the nearest whole of integer $\Delta c$ and $\Delta u$.

$$
\begin{aligned}
\widetilde{U} & =(\Delta \widetilde{u} \times \Delta \widetilde{c}) \circ \widetilde{R} \\
\mu_{\widetilde{U}}(z) & =\vee \mu_{\widetilde{R}}(x, y, z) \wedge\left[\mu_{\Delta \widetilde{u}}(x) \wedge \mu_{\Delta \widetilde{c}}(y)\right]
\end{aligned}
$$


The only obtained fuzzy vector above reflects a situation of the different fuzzy subset of $\Delta c, \Delta u$.Fuzzy issues should be considered because actually the charged object can only accept the control of a specific one.

There are many ways in the current ambiguity, such as the law of focus area, membership of the largest, and the weighted average method and so on.

The weighted average method is applied here, and precise control variables obtained, the following formula

$$
U^{*}=\frac{\sum \mu_{\widetilde{U}}\left(z_{i}\right) \times z_{i}}{\sum \mu_{\widetilde{U}}\left(z_{i}\right)}
$$

The control of variable $U^{*}$, which is obtained by the control table established on SCM and formatted with fuzzy and group switching capacitor guarantee load reactive power at the best value in the scope.

Fig. 5 is the Control variable value of $\Delta c$ and $\Delta u$ in $(1,4)$.

Their surface map is described by Fig. 6 .

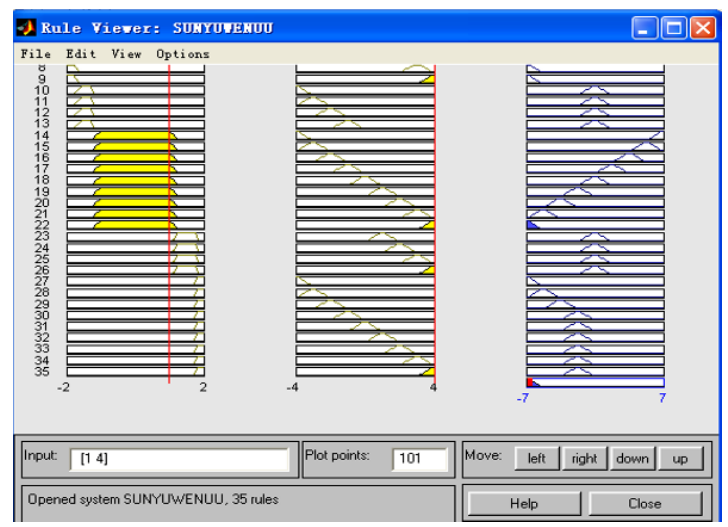

Fig. 5: Input/output variable getting value table

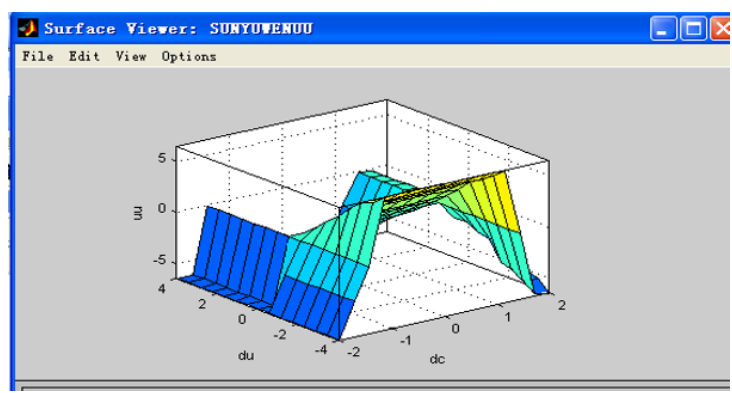

Fig.6: Input/output relation cambered face 


\section{CONCLUSION}

Reactive compensation has a very important practical significance and economic benefits. Grid voltage level is determined by the system-wide balance of reactive power, usually voltage and reactive power compensation is not a function of the relationship between the curve, and family of curves. The important feature of fuzzy logic control is simple, only a twodimensional logic form, that is the simulated experts operation (Guo Zongren et al., 2002).

Fuzzy reasoning simulation using MATLAB, with a voltage deviation $\Delta u$ and reactive power deviation $\Delta c$ as input, commonly controls switching capacitors, compensates for the inaccuracy the of the previous single factor control ,eliminates the unnecessary reciprocating switching, and improves the control strategy, and achieves the control of reactive power compensation based on fuzzy reasoning.

Obtaining the membership function of the voltage deviation and the reactive power deviation, the control of variable $U^{*}$, which is obtained by the control table established on SCM and formatted with fuzzy, and group switching capacitor guarantee load reactive power at the best value in the scope.

\section{ACKNOWLEDGEMENTS}

As with any effort for this paper, there are a number of people who contributed to this in a roundabout way. Without their help, this paper would not exist.

Special thanks to professor Yang Yong, who is my $\mathrm{PhD}$ supervisor not only suggested the whole structure of design, but who always gave me good idea, no matter how odd.

Particular thanks are due to Sun Yuwen, the teacher works in School of Information Science and Technology, Nanjing Agricultural University for sharing her knowledge and skills environment.

The author is also grateful to Dong Jianguo for his help, advice for mathematical modeling, comments, and excellent proofreading skills and gently showed me the errors in my ways.

Finally, the authors would like to thank the countless people who contributed to this paper with informal reviews and suggestions. 


\section{REFERENCES}

Cai Zhongqin, Guo Zhizhong. Expected model-based reactive moment method for distribution network reactive compensation, IEEE, 1998:1438-1442

E Zhijun, Fang Dazhong, Chan Kawing, Hybrid simulation algorithm based on SVC dynamic phasor model, Journal of Tianjin University (Science and Technology), 2008, 41(3):331337

E. M. John. Reactive compensation tutorial, IEEE Power Engineering Society Winter Meeting, Toronto, Canada, 2002:515-519

Gao Bo, Zhu Yubo. Dynamic reactive power compensation scheme for pumping units, Electric Age, 2003, 4:89-90

Guo Zongren et al. PLC application system design and communications network technology, Posts \& Telecom Press, 2002

Liu Liming, Liu Dichen, Shi Jin. Study on intelligent dynamic reactive power compensation device, Electric Power Automation Equipment, 2002, 22(8):28-31

Liu Shumin, Li Kuiwen, Liu Hui et al. Selection of reactive power compensation equipment, Electric Drive, 2002, 4:61-64

Liu Xiaochuan, Zhang Bin, Xu Zhihan. Integrate control system of voltage and reactive power based on fuzzy logic control theory, Electric Engineering, 2000, 6:4-7

Mao Meijun. A calculation example of reactive compensation optimization in power system, Zhejiang Electric Power, 2007, 26(1):73-74

P. Douglas, T. Dava. How to turn a substation into a database server, IEEE Computer Application in Power, April 1999:29-35

Tang Yong, Hou Junxian, Liu Wenzhou. The modeling of distribution network and var compensator and induction motor in the load model for power system digital simulation, Proceedings of the CSEE, 2005, 25(3): 8-12

Wang Chun, Cheng Haozhong, Chen Ken. Integrated Optimization Algorithm of Dynamic Reactive Power for Distribution System, Transactions of China Electrotechnical Society, 2008, 23(2):109-114

Wu Gongwen, Ai Qian. Configuration of reactive power compensation equipment, Low Voltage Apparatus, 2008, 4:51-53

Xiong Hugang, Cheng Haozhong, Zhang Jietan et al. Multi-objective reactive power compensation planning based on improving system reactive power reserve capacity, High Voltage Engineering, 2008, 34(2): 309-313, 318

$\mathrm{Xu}$ Yonghai, Jin Yan, Yao Baoqi et al, Simulation software of harmonic filter and reactive power compensation for distribution system, Electric Power Automation Equipment, 2006, 26(12):93-95

Yu Haisheng, Pan Songfeng. Measurement of electrical parameters based on complex sequence FFT and phase locking principle, Power System Technology, 2000, 24(3): 59-61 